COLLOQUE DE PHYSIQUE

Colloque C2, supplément au $\mathrm{n}^{\circ} 2$, Tome 51, Février 1990

ler Congrès Français d'Acoustique 1990

\title{
REFLECTION, BY A TWO-DIMENSIONAL OCEAN SUREACE, OF PLANE SOUND WAVES COMING FROM THE WATER SIDE
}

\author{
W.C. MEECHAM $(1)$ and W. - W. LIN *, (1) \\ Department of Mechanical, Aerospace, and Nuclear Engineering, School of \\ Engineering and Applied Science, University of California, Los Angeles, \\ California 90024-1597, U.S.A. \\ *National Sun Yat Sen University, Kaohsiung. Taiwan 800, Republic of \\ China
}

\begin{abstract}
Resumé - Des problèmes impliquant la réfiexion de radiation par une surface aléatoirement singulière se rencontrent dans de nombreuses applications. Nous serons tout d'abord intéressé par l'étude des ondes planes acoustiques qui se propagent sur la surface de l'eau. La variation de la pression acoustique disparait alors à la surface. Pou cela, nous utilisons un développement en fonctionnelles Gaussiènes. Cette méthode peux être étendue à des situations plus générales constituées par un ou plusieurs differents milieux séparés par des surfaces irrégulières en trois dimensions. Nous etudions dans le cadre de cet article une surface singuliere en deux dimensions avec ondle incidente. Avec l'aide du développement de Weiner-Hermite (W-H), nous representons le champs stochastique en utilisant la surface Gaussiène de réflexion comme premier élément du développement ainsi que troix termes supplémentaires. Des effets de réflexions multiples sont alors visibles, même dans le cas oū des termes d'ordre inférieur sont présents. De telles réflexions peuvent grandement entrainer des "réflexions" en retou pour des petits angles d'incidence.
\end{abstract}

\begin{abstract}
Problems involving the reflection of radiation by random rough surfaces arise in a number applications. We shall be interested primarily in an acoustic plane wave impinging on the surface from the water side. The acoustic pressure changes vanish at the surface. We use an expansion in polynomials of Guassian functionals. The method can be extended to more general situations involving more than one medium with irregular separating surfaces and three dimensional surfaces. We treat here a two-dimensional rough surface. By means of the Wiener-Hermite (W-H) expansion, we represent stochastic field functions using the (assumed) Gaussian reflecting surface as the basic element, to three terms. Multiple reflection effects are visible, even for these low order terms in the expansion. Such reflections can be expected to greatly enchance backscatter at grazing incidence.
\end{abstract}

\section{1 - INTRODUCTION}

The literature involving reflections from rough surfaces is enormous. We shall not attempt to review that work; for a start the reader can refer to ref. 1 . Methods for the treatment of problems considered here may be approximately divided into two groups, low frequency and high frequency. Intermediate frequencies, most likely must be treated numerically. Regrettably many of the methods available for scattering from rough surfaces must be applied to but one such surface, or to a very special class of such surfaces. We very often need reflections from ensembles of surfaces, so that non-stochastic methods must be used once for each member of the ensemble and the results averaged.

For low frequencies a perturbation treatment is appropriate. The main criterion for validity is that rms surface deviations, from the average plane, be small compared with the sound wavelength. It has often been true that perturbation treatments are confined to the first order term, that is to terms proportional to the surface displacement. Though such first-order treatments are useful, they cannot in their nature include multiple reflections. This latter defect means that for such problems, as for example trying. to determine back scatter for low angle incidence, we expect predicted back scatter to be considerably below actual values, because the back scatter is then strongly influenced by multiple reflections. It will be seen that the W-H stochastic expansion permits many high order perturbation terms (contributing to high order multiple scatterings) to be included in low order terms. It must be emphasized that the W-H expansion is much more than a power series expansion. For example consider $e^{i k \xi(x)}$ where $\xi(\mathrm{x})$ is the Gaussian surface (ensemble). The zeroth order term in W-H expansion is $\mathrm{e}^{-1 / 2 \mathrm{k}^{2} \xi_{0}^{2}}$ where $\xi_{0}^{2}$ is the variance. The corresponding zeroth order term in the perturbation expansion is 1 . We discuss this below. The W-H procedure is not restricted to moments but is an expansion of the stochastic process, here the reflected field, in terms of polynomials in a Gaussian process. This representation has proved very useful in the statistical theory of turbulence $/ 2 /$. We have obtained encouraging preliminary results using the W-H expansion on reflection problems $/ 3 /$.

\footnotetext{
(1) Partially supported by the Office of Naval Research, Department of Navy, U.S.A.
} 
For high frequencies one usually uses a physical optics treatment employing the Kirchhoff approximation. The normal derivative of the surface pressure is assumed then to be twice that of the incident field. Then Huygens' principal expresses the reflected field in terms of this normal derivative on the surface (the field itself vanishes). The main criterion for validity is that the sound wavelength should be small compared to typical radii of curvature of the reflecting surface.

Below we express the reflected field in terms of downward propagating waves the Rayleigh hypothesis. There are regions in a quite irregular surface where one might expect that the reflected field would consist as well of, if not upward propagating planewaves, at least portions thereof, see Fig. 1. There have been recent proposals that in a certain sense only downward waves may be needed $/ 4 /$.

\section{WIENER-HERMITE EXPANSION}

Consider single frequency sound impinging on a two dimensional ocean surface, from the water side. The boundary condition is that the pressure change vanish at the surface. We use the W-H representation $/ 2 \%$. Assume that the rough surface is statistically homogeneous and Gaussian with zero mean and with a variance of $\xi_{0}^{2}$. Represent the normalized, incident field by [suppressing a time factor $\exp (-\mathrm{i} \omega \mathrm{t})$ ]

$$
\mathrm{p}_{\mathrm{i}}=\exp \left[\mathrm{i} \mathrm{k}_{\mathrm{xi}} \mathrm{x}+\mathrm{i}\left(\mathrm{k}^{2}-\mathrm{k}_{\mathrm{xi}}^{2}\right)^{1 / 2} \mathrm{z}\right]
$$

and the reflected field by

$$
\mathrm{p}_{\mathrm{r}}=\int_{-\infty}^{\infty} \alpha\left(\mathrm{k}_{\mathrm{x}}\right) \exp \left[\mathrm{i} \mathrm{k}_{\mathrm{x}} \mathrm{x}-\mathrm{i}\left(\mathrm{k}^{2}-\mathrm{k}_{\mathrm{x}}^{2}\right)^{1 / 2} \mathrm{z}\right] \mathrm{dk} \mathrm{x}
$$

both solutions of the Helmholtz equation with $\mathrm{k}$ the wave number. There is an implicit assumption here that the troughs are not too deep, to avoid the Rayleigh error (if such exists). We have $k_{x i}=k$ sin $\Theta_{i}$ and $\alpha$ is the reflection coefficient. When $k_{x}>k$ choose the positive root in Eq. (1) so the waves damp out exponentially away from the surface, $z$ positive upward. The boundary condition gives at $z=\xi(x)$,

$$
\begin{aligned}
& \int_{-\infty}^{\infty} \alpha\left(k_{\mathrm{x}}\right) \exp \left[\mathrm{i} \mathrm{k}_{\mathrm{x}} \mathrm{x}-\mathrm{i}\left(\mathrm{k}^{2}-\mathrm{k}_{\mathrm{xi}}^{2}\right)^{1 / 2} \xi(\mathrm{x})\right] \mathrm{dk_{ \textrm {x } }} \\
& =-\exp \left[\mathrm{i} \mathrm{k}_{\mathrm{xi}} \mathrm{x}+\mathrm{i}\left(\mathrm{k}^{2}-\mathrm{k}_{\mathrm{xi}}^{2}\right)^{1 / 2} \xi(\mathrm{x})\right]
\end{aligned}
$$

where the ensemble of ocean surfaces is described by $z=\xi(x)$.

The generalized $\mathrm{W}-\mathrm{H}$ polynomials (functionals) are $/ 2 /$

$$
\begin{aligned}
& \mathrm{H}^{(0)}(0)=1 \\
& \mathrm{H}^{(1)}(\mathrm{x})=\xi(\mathrm{x}) \\
& \mathrm{H}^{(2)}\left(\mathrm{x}_{1}, \mathrm{x}_{2}\right)=\xi\left(\mathrm{x}_{1}\right) \xi\left(\mathrm{x}_{2}\right)-\mathrm{R}\left(\mathrm{x}_{1}-\mathrm{x}_{2}\right) \\
& \vdots
\end{aligned}
$$

and are constructed so as to be statistically orthogonal,

$$
\left\langle\mathrm{H}^{(\mathrm{m})}(\xi) \mathrm{H}^{(\mathrm{n})}(\xi)>=0, \quad \mathrm{~m} \neq \mathrm{n}\right.
$$

and $R(x)$ is the autocorrelation of $\xi(x)$.

We must solve for $\alpha$, needed in (2), using (3). Expand the exponentials in (3) and let the successive W-H terms for $\alpha$ be given by, through four terms,

$$
\alpha(\mathrm{k})=\alpha^{(0)}(\mathrm{k})+\alpha^{(1)}(\mathrm{k})+\alpha^{(2)}(\mathrm{k})+\alpha^{(3)}(\mathrm{k})+\ldots
$$

with $\alpha^{(n)}(k)$ Fourier Transforms of linear combinations of $H^{(n)} / 2,3 /$. The governing (coupled) equations for $\alpha^{(n)}$ are obtained by multiplying the W-H representation, (3), (6) successively by $\mathrm{H}^{(0)}, \mathrm{H}^{(\mathrm{n})}, \mathrm{H}^{(2)}$ etc. and averaging, using the orthogonality relation (5).

After some manipulation, we find the zeroth order reflection coefficient,

$$
\alpha_{3}^{(0)}\left(k_{x}\right)=-2 k \cos \Theta_{i} e_{3}\left(k \sin \Theta_{i}\right) \delta\left(k_{x}-k \sin \Theta_{i}\right) \cdot\left[1+k \cos \Theta_{i} e_{4}\left(k \sin \Theta_{i}\right)\right]^{-1}
$$

where the subscript 3 indicates that we use just three terms and $\Theta_{i}$ is the incident angle measured from the normal of the mean.

The first order reflection coefficient is 


$$
\begin{gathered}
\alpha_{3}^{(1)}\left(k_{x}\right)=\left(-i k \cos \Theta_{i}\right) \xi\left(k \sin \Theta_{i}-k_{x}\right) \cdot \exp \left[-1 / 2\left(k_{x}^{2}-k^{2} \sin ^{2} \Theta_{i}\right) \xi_{0}^{2}\right] \cdot\left\{1 /\left[1+k \cos \Theta_{i} e\left(k \sin \Theta_{i}\right)\right] \pi\right\} \\
\cdot\left\{1-k \cos \Theta_{i} e_{3}\left(k \sin \Theta_{i}\right) \cdot\left[1+k \cos \Theta_{i} e\left(k_{x i}\right) /\left[1+k \cos \Theta_{i} e_{4}\left(k_{x i}\right)\right]\right\}\right.
\end{gathered}
$$

where $\xi\left(k_{x}\right)$ is the Fourier Transform of $\xi(x)$.

$$
\begin{aligned}
& \text { The } \alpha^{(2)}\left(k_{\mathrm{x}}\right) \text { can be found } \\
& \begin{array}{l}
\alpha_{3}^{(2)}\left(\mathrm{k}_{\mathrm{x}}\right)=\exp \left[-1 / 2 \mathrm{k}_{\mathrm{x}}^{2} \xi_{0}^{2}+\mathrm{k}^{2} \sin ^{2} \Theta_{\mathrm{i}} \xi_{0}^{2}\right]\left[\left(\mathrm{k} \cos \Theta_{\mathrm{i}}\right) /(2 \pi)\right]^{2} \cdot \int d \mathrm{k}_{\mathrm{x}}^{\prime} \mathrm{H}_{\xi}^{(2)}\left(\mathrm{k} \sin \Theta_{\mathrm{i}}-\mathrm{k}_{\mathrm{x}}-\mathrm{k}_{\mathrm{x}}^{\prime}, \mathrm{k}_{\mathrm{x}}^{\prime}\right) \\
\cdot 2\left[\mathrm{k}^{2}-\left(\mathrm{k}_{\mathrm{x}}+\mathrm{k}_{\mathrm{x}}^{\prime}\right)^{2}\right]^{1 / 2} \cdot\left\{1+\left[\mathrm{k}^{2}-\left(\mathrm{k}_{\mathrm{x}}+\mathrm{k}_{\mathrm{x}}^{\prime}\right)^{2}\right]^{1 / 2} \cdot \mathrm{e}\left(\mathrm{k}_{\mathrm{x}}+\mathrm{k}_{\mathrm{x}}^{\prime}\right)\right\}^{-1} \cdot\left\{1-\mathrm{k} \cos \Theta_{\mathrm{i}}\left[1+\mathrm{k} \cos \Theta_{\mathrm{i}} \mathrm{e}\left(\mathrm{k} \sin \Theta_{\mathrm{i}}\right)\right]\right. \\
\left.\mathrm{e}_{3}\left(\mathrm{k} \sin \Theta_{\mathrm{i}}\right) /\left[1+\mathrm{k} \cos \Theta_{\mathrm{i}} \mathrm{e}_{4}\left(\mathrm{k} \sin \Theta_{\mathrm{i}}\right)\right]+\mathrm{k}^{2} \cos ^{2} \Theta_{\mathrm{i}} \mathrm{e}_{3}\left(\mathrm{k} \sin \Theta_{\mathrm{i}}\right) /\left[1+\mathrm{k} \cos \Theta_{\mathrm{i}} \mathrm{e}_{4}\left(\mathrm{k} \sin \Theta_{\mathrm{i}}\right)\right]\right\}
\end{array}
\end{aligned}
$$

where we define the functions

$$
\begin{aligned}
& e\left(k^{\prime}\right)=\int E\left(k^{\prime}-k_{x}\right)\left(k^{2}-k_{x}^{2}\right)^{1 / 2} d k_{x} \quad e_{2}\left(k^{\prime}\right)=\int E\left(k^{\prime}-k_{x}\right)\left(k^{2}-k_{x}^{2}\right) d k_{x} \\
& e_{3}\left(k^{\prime}\right)=\int E\left(k^{\prime}-k_{x}\right)\left(k^{2}-k_{x}^{2}\right)^{1 / 2}\left[1+e_{2}\left(k_{x}\right)\right] \cdot\left[1+e_{2}\left(k_{x}\right)\right] /\left[1+\left(k^{2}-k_{x}^{2}\right)^{1 / 2} e\left(k_{x}\right)\right] d k_{x} \\
& e_{4}\left(k^{\prime}\right)=\int E\left(k^{\prime}-k_{x}\right)\left(k^{2}-k_{x}^{2}\right)^{1 / 2}\left[1+e_{2}\left(k_{x}\right)\right] \cdot\left[1+k \cos \Theta_{i} e\left(k_{x}\right)\right] /\left[1+\left(k^{2}-k_{x}^{2}\right)^{1 / 2} e\left(k_{x}\right)\right] d k_{x}
\end{aligned}
$$

Note that the procedure allows an explicit solution for $\alpha^{(i)}$.

\subsection{Conservation of Acoustic Energy}

The conservation law for intensity in terms of $\mathrm{W}-\mathrm{H}$ coefficients is

$$
k \cos \Theta_{\mathrm{i}}=k \cos \Theta_{\mathrm{i}}\left|-1+\alpha^{(0)}\left(\mathrm{k}_{\mathrm{x}}\right)\right|^{2}+(2 \pi / 2 \mathrm{~L}) \sum_{1}^{\infty} \int_{-\infty}^{+\infty}\left|\alpha^{(\mathrm{n})}\left(\mathrm{k}_{\mathrm{x}}\right)\right|^{2}\left(\mathrm{k}^{2}-\mathrm{k}_{\mathrm{x}}^{2}\right)^{1 / 2} \mathrm{dk} \mathrm{x}
$$

where $2 \mathrm{~L}$ is the (large) size of the radiated ocean surface. The left hand side is the normalized incident acoustic power falling on a unit area. The first term on the right hand side represents the specularly reflected power whereas the series expansion represents the nonspecularly scattered power per unit area.

The incident plane wave can be scattered by a rough surface into various directions. If we denote $s\left(\Theta / \Theta_{\mathrm{i}}\right)$ as the average power scattered into the angle direction $\Theta$ with incident angle $\Theta_{i}$, then

$$
\mathrm{S}\left(\Theta \mid \Theta_{\mathrm{i}}\right) \cos \Theta \mathrm{d} \Theta=(1 / \mathrm{k})(2 \pi / 2 \mathrm{~L}) \cdot \sum_{1}^{\infty}<\left|\alpha\left(\mathrm{k}_{\mathrm{x}}\right)\right|^{2}>\left(\mathrm{k}^{2}-\mathrm{k}_{\mathrm{x}}^{2}\right)^{1 / 2} \mathrm{dk}_{\mathrm{x}}
$$

\section{DISCUSSION OF RESULTS AND CONCLUSIONS}

Some results for a water surface with energy spectrum

$$
E\left(k^{\prime}\right)=\left[k^{\prime} \pi^{1 / 2}\right]^{-1} \exp \left[-\left(k^{\prime} / K\right)^{2}\right]
$$

( $K$ is the characteristic wave number of water waves) are given. Figure 2 shows that three terms (for $k / K=100$ ) of the W-H expansion give energy conservation to considerably higher sound frequencies than does the perturbation treatment (which uses the same number of terms). The sound frequency is $f=k c_{0} /(2 \pi)$, with $c_{o}$ the speed of sound.

There is a reduction in the specular reflection ( $\delta$-function in angle) caused by the rough surface. The nonspecular portions are given in Figs. 3 -4.

Figure 4 , for $\Theta_{i}=60 \mathrm{deg}$, shows evidence of multiple scattering. For incident radiation nearer grazing incidence, Fig. 1, surface slopes which increase the angle of the reflected radiation from the normal tend to be shadowed, thus favoring throwing the reflected radiation back nearer to the incident direction. We see this effect in Fig. 4 for $(\mathrm{k} / \mathrm{K})=1$. As $\mathrm{k} / \mathrm{K}$ increases, $\mathrm{k}, \xi_{0}$ constant, surface slopes decrease and the radiation tends to approach specular reflection.

We have also calculated the reflecting properties of the preferred water surface energy spectrum function $/ 5 / 8$ )

$$
\mathrm{E}(\mathrm{k})=2 \xi_{0}^{2} \mathrm{~K}^{4} /\left[\pi\left(\mathrm{k}^{2}+\mathrm{K}^{2}\right)^{5}\right]^{1 / 2} \quad \mathrm{k} \xi_{0}=\pi / 10
$$

In Fig. 5 we see the energy reflection characteristics for $\mathrm{K} / \mathrm{k}=2$, which can be compared with scattered radiation from the Gaussian correlation shown above, first of all it reduces as we approach grazing; that is, most of the energy returns to the specular portion of the field. Further, again, the angle of maximum radiation shifts toward the incident direction. angle but at angles considerably less as in Fig. 4. 
The slow, evanescent surface waves are of interest in many applications. in Fig. 6 we show for an energy spectrum similar to Eq. (17) the average energy contained in these slow waves plotted against $\Theta_{i}$. All of these values have been normalized using the incident flux, and $k \xi_{0}=\pi / 10$. We see that there is relatively little energy flux (flux of course for these waves being entirely parallel to the surface). As the sound wavelength gets larger, we see even less energy being carried parallel to the surface.

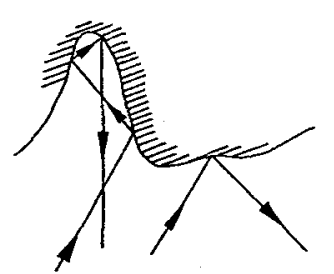

Figure 1: Single scattering and multiple scattering for different surface wavelengths. (a) For long surface wavelength, only single scattering occurs. (b) For short surface wavelength, there is some multiple scattering. Note the tendency to increase the backward scattered portion (see text).

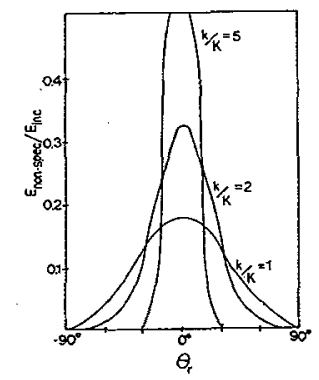

Figure 3: Nonspecular reflection portion for normal incidence; $\mathrm{k} \xi_{0}=\pi / 10$ for all cases.

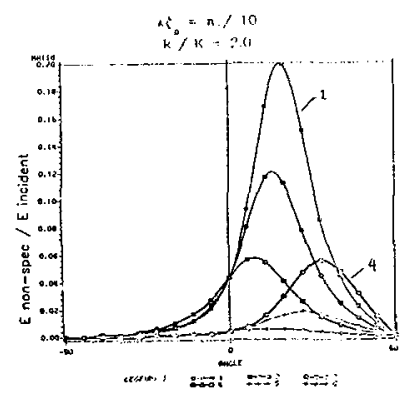

Figure 5: Angular distribution of nonspecular scattering for an experiment spectrum surface. In this figure, 1: $\psi=0$ for $\theta_{i}=30$, and $4: \theta_{i}=60$, other curves are for scattering out the plane.

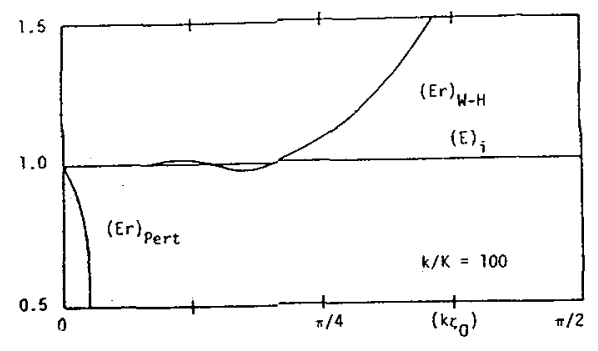

Figure 2: Energy conservation check for Gaussian surface-spectrum, normal incidence. Here $\left(\mathrm{E}_{\mathrm{T}}\right)_{\text {pert }}$ is the reflected energy from a perturbation treatment, same number of terms, $\left(\mathrm{E}_{\mathrm{r}}\right)_{\mathrm{W}-\mathrm{H}}$ by the $\mathrm{W}-\mathrm{H}$ procedure.

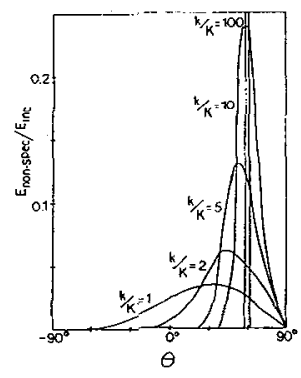

Figure 4: Nonspecular reflection portion for oblique incidence; $k \xi_{0}=\pi / 10$ and $\theta_{i}=60 \mathrm{deg}$ for all cases.

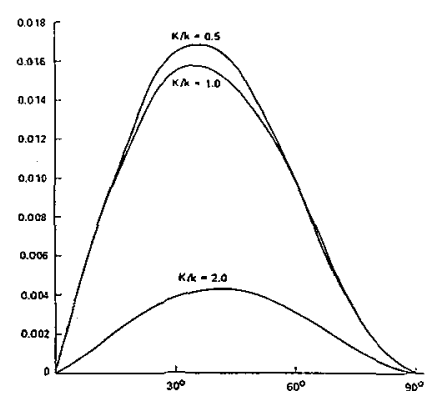

Figure 6: The average energy flux contained in the slow, surface wave. Energy fluxes are normalized by the incident flux, $k \zeta_{0}=\pi / 10$.

\section{REFERENCES}

11/. Beckman, P. and Spizzicino, A., "Scattering of Electromagnetic Waves from Rough Surfaces", Pergamon Press, N.Y. (1953).

12\%. Hogge, H.D. and Meecham, W.C., J. Fluid Mech., 85, (1978) 325.

131. Lin, W.W., Ph.D. Thesis, UCLA School of Engineering and Applied Science. 1986.

14f Milder, M., Arete Associates, Los Angeles, CA, Private Communication.

15/ Phillips, O.M.l, "The Dynamics of the Upper Ocean," Cambridge University Press (1966). 